\title{
Evaluation of commonness of earhead caterpillars by changing sowing dates in $k$ harif Sorghum
}

\author{
B. Kiran Gandhi ${ }^{1}$, Shekharappa ${ }^{2}$ \\ ${ }^{I}$ (Agriculture Entomology, University of Agricultural Sciences Dharwad, India) \\ ${ }^{2}$ (Agriculture Entomology, University of Agricultural Sciences Dharwad, India)
}

\begin{abstract}
Sorghum hybrids like CSH-14 and CSH-16 were sown on different dates with weekly interval. Lowest larval population was recorded at flowering stage (1.16 larvaelearhead), when crop was sown during $12^{\text {th }}$ and 19 ${ }^{\text {th }}$ June 2010. CSH-14 hybrid recorded (2.00 larvaelearhead) low larval load on $12^{\text {th }}$ June, 2010 date of sowing. Per cent earhead damage was minimum (11.33\%) and maximum grain yield was recorded (39.81 q/ha) when CSH-14 was sown on $12^{\text {th }}$ June 2010. As sowings were delayed larval count and per cent earhead damage increased whereas grain yield decreased.
\end{abstract}

Keywords - earhead, grain, hybrid, sown, yield

\section{Introduction}

In India, sorghum ranks third in area and production after rice and wheat, covering an area of 7.68 mha. producing 7.31 million tones with an average yield of $9.52 \mathrm{q}$ per ha, Anonymous ${ }^{[1]}$. Several reasons have been attributed for the low yield of sorghum. Among them insect pests ravage is one of the principal factor. Among different insect pests of sorghum, the ear head caterpillars viz., Helicoverpa armigera (Hubner), Cryptoblabes gridiella (Miller) and Euproctis subnotata (Walker) and Stenochroia elongella (Miller) are important species. Helicoverpa armigera is one of the most important earhead pest reported to cause as much as 37.11 per cent yield loss in sorghum, Kulkarni et al. ${ }^{[2]}$.Euproctis subnotata is principally a pest of sorghum. It assumed the importance of a serious pest in recent years particularly with the introduction of hybrid sorghums, Patil ${ }^{[3]}$. Cryptoblabes gnidiella causes considerable loss to hybrid sorghum, the larval feed on milky and hardy grains inside the earheads, Srivastava and Singh ${ }^{[4]}$. Although, the larvae of the old world webworm, Stenachroia elongella is smaller in size than Heliothis, probably causes more damage.

\section{Methodology}

The experiment included seven treatments and three replications. The different treatments included sowing dates on different weeks viz., June $2^{\text {nd }}$, June $3^{\text {rd }}$, June $4^{\text {th }}$, July $1^{\text {st }}$, July $2^{\text {nd }}$, July $3^{\text {rd }}$, July $4^{\text {th }}$ weeks. CSH16 was used in the experimentation and each plot size of $4 \times 2.7 \mathrm{~m}$. The observation were made on the number of larvae on 25 sorghum earheads at weekly interval from flowering to till harvest and expressed as number of larvae per earhead. Though, the dates of sowing were planned during $3^{\text {rd }}$ and $4^{\text {th }}$ weeks of May and $1^{\text {st }}$ week of June but could not be done because of non-receipt of rains. Observations were recorded on number of larvae per earhead, per cent earhead Damage And Grain Yield.

\subsection{Results}

\section{Number of larvae per earhead}

\subsubsection{Earhead caterpillars incidence on different stages of earhead}

Among different earhead caterpillars, H. armigera was major and E. subnotata and S. elongella were minor ones on both the popular hybrids (CSH-14 and CSH-16). With irrespective of hybrids, on flowering stage least larval load was recorded (1.16 larvae/earhead) when crop was sown early i.e., both $12^{\text {th }}$ June $\left(\mathrm{D}_{1}\right)$ and $19^{\text {th }}$ June $\left(D_{2}\right)$ 2010. On milk stage larval load ranged from 4.33 larvae per earhead $\left(D_{1}\right)$ to 8.00 larvae per earhead $\left(D_{3}\right)$. However, all these treatments were found on par with each other. At dough stage, larval population ranged from $1.16\left(\mathrm{D}_{3}\right)$ to $4.50\left(\mathrm{D}_{6}\right)$ larvae per earhead. However, there was no significant difference existed among treatments (TABLE 1). Amongst different stages, dough stage recorded lowest mean number of larvae $(2.28 \%)$ followed by flowering (3.14 larvae/earhead) and milk stage (6.52 larvae/earhead) irrespective of different dates of sowing. It indicated that dough stage showed moderate resistance to earhead caterpillars as compared to flowering and milk stages.

\subsubsection{Earhead caterpillars incidence on hybrids}

CSH-14 hybrid recorded 3.81 larvae per earhead, as against CSH-16 which recorded (4.15 larvae/earhead) with irrespective of different dates of sowing. This clearly indicated superiority of CSH-14 over 
CSH-16 establishing moderate resistance as it was possessing loose earhead. Mean larval number recorded more than economic threshold level during different dates of sowing, on all the stages and in two different hybrids.

\subsection{Discussion}

Kongwad ${ }^{[5]}$ and Shivanand ${ }^{[6]}$ carried experiment in Dharwad, India. Reported occurrence of earhead caterpillars were confined to kharif season. Incidence of earhead caterpillars was more on the crop sown during June second fortnight compared to the crop sown on July first fortnight. Amongst all stages with irrespective of hybrids on milk stage registered maximum larval population (6.52 larvae/earhead) when compared to other two stages and all these stages are significantly differed with each other. Among CSH-14 and CSH-16 although CSH-14 recorded less infestation (3.81 larvae/earhead) but statistically found on par with CSH-16 with respect to larval incidence

\subsection{Results}

\section{Per cent earhead damage}

Per cent earhead damage was mainly due to Helicoverpa armigera (Hubner) and to little extent by Euproctis subnotata (Walker) and Stenachroia elongella (Miller). Mean per cent earhead damage with irrespective of hybrids on different dates of sowing ranged from 13.33 to 44.0 per cent. Minimum mean per cent earhead damage $(13.33 \%)$ recorded on $12^{\text {th }}$ June $2010\left(D_{1}\right)$ was on par with $19^{\text {th }}$ June $2010\left(D_{2}\right)$ recorded 16.66 per cent and significantly differed with other treatments. Amongst hybrids, irrespective of different dates of sowing CSH-14 recorded minimum mean per cent earhead damage $(28.76 \%)$ which was on par with CSH-16 which recorded 31.28 per cent (TABLE 2). In CSH-16 hybrid, minimum mean per cent earhead damage was recorded $(15.33 \%)$ when the crop was sown on $12^{\text {th }}$ June $2010\left(D_{1}\right)$ which was on par with $D_{2}\left(19^{\text {th }}\right.$ June, 2010$)$ recorded 17.00 per cent and differed significantly with all other treatments. Minimum mean per cent earhead $(11.33 \%)$ damage was recorded from $12^{\text {th }}$ June $2010\left(D_{1}\right)$ sowing in CSH-14. All the treatments differed significantly with each other. At all dates of sowings in two hybrids CSH-14 and CSH-16, per cent earhead damage crossed economic threshold level i.e., more than 10 per cent this showed that manipulation of sowing date of escape the attack from earhead caterpillars alone will not be helpful to the crop from earhead caterpillar.

\section{Grain yield}

Mean grain yield with irrespective of hybrids on different dates of sowing ranged from 9.98 to $39.39 \mathrm{q}$ per ha. Maximum grain yield of $39.39 \mathrm{q}$ per ha was recorded when crop sown early on $19^{\text {th }}$ June, $2010\left(\mathrm{D}_{1}\right)$. Amongst hybrids, CSH-16 recorded maximum mean grain yield of 28.68 q per ha whereas CSH-14 registered $26.14 \mathrm{q}$ per ha. However, they were found on par with each other (TABLE 3).

\section{Conclusion}

CSH-14 hybrid recorded (2.00 larvae/earhead) low larval load, minimum per cent earhead damage $(11.33 \%)$ and maximum grain yield $(39.81 \mathrm{q} / \mathrm{ha})$ when early sowing was taken on $12^{\text {th }}$ June 2010 . As sowings were delayed larval count and per cent earhead damage increased whereas grain yield decreased.

\section{References}

[1] Anonymous, Agricultural Statistics at a Glance, Directorate of Economics and Statistics, 2009, 90-92.

[2] Kulkarni, K. A, Thontadharya, T. S, Jotwani, M. G, and Parameshwarappa, R, Present status of earhead caterpillars on sorghum and their management. Presented at the All India Sorghum Workshop, Coimbatore, India, 12-14 May, 1980.

[3] Patil, S. B, Studies on the biology and control of tussock caterpillar, Euproctis subnotata Walker (Lepidopera : Lymantriidae) on sorghum, M. Sc. (Agri.) Thesis, University of Agricultural Sciences Dharwad, Karnataka, India, 1985

[4] Srivastava, A. S. and Singh, Y. P, Control of Heliothis armigera Hub. (Noctuidae : Lepidoptera) attacking sorghum. Curr. Sci., 42, $1973,865-867$

[5] Kongwad, S. B, Bio-ecology and management of sorghum earhead caterpillar with special reference to Cryptoblabes gnidiella (miller), M. Sc. (Agri.) Thesis, University of Agricultural Sciences Dharwad, Karnataka, India, 2000.

[6] Shivanand, Bio-ecological management of Helicoverpa armigera (Hub.) on sorghum, M. Sc. (Agri.) Thesis, University of Agricultural Sciences Dharwad, Karnataka, India, 2002. 
Evaluation of commonness of earhead caterpillars by changing sowing dates in kharif Sorghum

Table 1: Effects of dates of sowing on the incidence of earhead caterpillar, Helicoverpa armigera at different stages on kharif sorghum

\begin{tabular}{|c|c|c|c|c|c|c|}
\hline \multirow{3}{*}{ Dates Of Sowing } & \multicolumn{5}{|c|}{ Number Of Larvae Per Earhead } & \multirow{3}{*}{ Mean } \\
\hline & \multicolumn{3}{|c|}{ Stages } & \multicolumn{2}{|c|}{ Hybrids } & \\
\hline & Flowering & Milk & Dough & CSH-16 & CSH-14 & \\
\hline$D_{1}-12^{\text {th }}$ June 2010 & $\begin{array}{c}1.16 \\
(1.47) \\
\end{array}$ & $\begin{array}{l}4.33 \\
(2.3) \\
\end{array}$ & $\begin{array}{c}1.33 \\
(1.53) \\
\end{array}$ & $\begin{array}{c}2.55 \\
(1.88) \\
\end{array}$ & $\begin{array}{c}2.00 \\
(1.73) \\
\end{array}$ & $\begin{array}{c}2.27 \\
(1.80)\end{array}$ \\
\hline$D_{2}-19^{\text {th }}$ June 2010 & $\begin{array}{c}1.16 \\
(1.47) \\
\end{array}$ & $\begin{array}{l}4.83 \\
(2.4) \\
\end{array}$ & $\begin{array}{c}2.16 \\
(1.78) \\
\end{array}$ & $\begin{array}{c}2.88 \\
(1.97) \\
\end{array}$ & $\begin{array}{c}2.55 \\
(1.88) \\
\end{array}$ & $\begin{array}{c}2.72 \\
(1.92)\end{array}$ \\
\hline$D_{3}-27^{\text {th }}$ June 2010 & $\begin{array}{c}4.66 \\
(2.38) \\
\end{array}$ & $\begin{array}{l}8.00 \\
(3.0) \\
\end{array}$ & $\begin{array}{c}1.16 \\
(1.47) \\
\end{array}$ & $\begin{array}{c}4.55 \\
(2.36) \\
\end{array}$ & $\begin{array}{c}4.66 \\
(2.38) \\
\end{array}$ & $\begin{array}{c}4.61 \\
(2.36) \\
\end{array}$ \\
\hline $\mathrm{D}_{4}-03^{\text {rd }}$ July 2010 & $\begin{array}{c}3.16 \\
(2.04) \\
\end{array}$ & $\begin{array}{l}7.16 \\
(2.8) \\
\end{array}$ & $\begin{array}{c}2.00 \\
(1.73) \\
\end{array}$ & $\begin{array}{c}4.33 \\
(2.31) \\
\end{array}$ & $\begin{array}{c}3.88 \\
(2.21) \\
\end{array}$ & $\begin{array}{c}4.11 \\
(2.26) \\
\end{array}$ \\
\hline $\mathrm{D}_{5}-10^{\text {th }}$ July 2010 & $\begin{array}{c}3.00 \\
(2.00) \\
\end{array}$ & $\begin{array}{l}6.83 \\
(2.8) \\
\end{array}$ & $\begin{array}{c}1.66 \\
(1.63) \\
\end{array}$ & $\begin{array}{c}4.11 \\
(2.26) \\
\end{array}$ & $\begin{array}{c}3.55 \\
(2.13) \\
\end{array}$ & $\begin{array}{c}3.83 \\
(2.19) \\
\end{array}$ \\
\hline $\mathrm{D}_{6}-17^{\text {th }}$ July 2010 & $\begin{array}{c}4.16 \\
(2.27) \\
\end{array}$ & $\begin{array}{l}7.50 \\
(2.9)\end{array}$ & $\begin{array}{c}4.50 \\
(2.35) \\
\end{array}$ & $\begin{array}{c}5.55 \\
(2.56) \\
\end{array}$ & $\begin{array}{c}5.22 \\
(2.49)\end{array}$ & $\begin{array}{c}5.38 \\
(2.52) \\
\end{array}$ \\
\hline $\mathrm{D}_{7}-24^{\text {th }}$ July 2010 & $\begin{array}{c}4.66 \\
(2.38)\end{array}$ & $\begin{array}{l}7.00 \\
(2.8)\end{array}$ & $\begin{array}{c}3.16 \\
(2.04) \\
\end{array}$ & $\begin{array}{c}5.11 \\
(2.47)\end{array}$ & $\begin{array}{c}4.77 \\
(2.40)\end{array}$ & $\begin{array}{c}4.94 \\
(2.43)\end{array}$ \\
\hline \multirow[t]{2}{*}{ Mean } & $\begin{array}{c}3.14 \\
(2.03)\end{array}$ & $\begin{array}{c}6.52 \\
(2.74)\end{array}$ & $\begin{array}{c}2.28 \\
(1.81)\end{array}$ & $\begin{array}{c}4.15 \\
(2.26)\end{array}$ & $\begin{array}{c}3.81 \\
(2.19)\end{array}$ & \\
\hline & \multicolumn{3}{|c|}{ S.Em. \pm} & \multicolumn{3}{|c|}{ C.D. At $5 \%$} \\
\hline Sowing Dates (D) & \multicolumn{3}{|c|}{0.32} & \multicolumn{3}{|c|}{0.89} \\
\hline Hybrids $(\mathrm{H})$ & \multicolumn{3}{|c|}{0.17} & \multicolumn{3}{|c|}{0.47} \\
\hline Stages $(\mathrm{S})$ & \multicolumn{3}{|c|}{0.21} & \multicolumn{3}{|c|}{0.58} \\
\hline Interaction $(\mathrm{D} \times \mathrm{S})$ & \multicolumn{3}{|c|}{0.56} & \multicolumn{3}{|c|}{1.56} \\
\hline Interaction $(\mathrm{D} \times \mathrm{H})$ & \multicolumn{3}{|c|}{0.45} & \multicolumn{3}{|c|}{1.26} \\
\hline
\end{tabular}

Figures in parentheses indicate square root $(\sqrt{\mathrm{x}+1})$ transformed values

Table 2: Effects of dates of sowing on the incidence of earhead caterpillar, Helicoverpa armigera on kharif sorghum

\begin{tabular}{|c|c|c|c|}
\hline \multirow{2}{*}{ Dates of sowing } & \multicolumn{3}{|c|}{ Per cent earhead damage } \\
\hline & CSH-16 & CSH-14 & Mean \\
\hline$D_{1}-12^{\text {th }}$ June 2010 & $\begin{array}{c}15.33 \\
(23.04)\end{array}$ & $\begin{array}{c}11.33 \\
(19.66)\end{array}$ & $\begin{array}{c}13.33 \\
(21.40)\end{array}$ \\
\hline $\mathrm{D}_{2}-19^{\text {th }}$ June 2010 & $\begin{array}{c}17.00 \\
(24.34) \\
\end{array}$ & $\begin{array}{c}16.33 \\
(23.82) \\
\end{array}$ & $\begin{array}{c}16.66 \\
(24.08) \\
\end{array}$ \\
\hline $\mathrm{D}_{3}-27^{\text {th }}$ June 2010 & $\begin{array}{c}34.66 \\
(36.05) \\
\end{array}$ & $\begin{array}{c}37.33 \\
(37.64) \\
\end{array}$ & $\begin{array}{c}36.00 \\
(36.85) \\
\end{array}$ \\
\hline $\mathrm{D}_{4}-03^{\text {rd }}$ July 2010 & $\begin{array}{c}33.66 \\
(35.45) \\
\end{array}$ & $\begin{array}{c}28.66 \\
(32.35) \\
\end{array}$ & $\begin{array}{c}31.16 \\
(33.91) \\
\end{array}$ \\
\hline $\mathrm{D}_{5}-10^{\text {th }}$ July 2010 & $\begin{array}{c}31.66 \\
(34.23) \\
\end{array}$ & $\begin{array}{c}26.00 \\
(30.64) \\
\end{array}$ & $\begin{array}{c}28.83 \\
(32.46) \\
\end{array}$ \\
\hline $\mathrm{D}_{6}-17^{\text {th }}$ July 2010 & $\begin{array}{c}41.66 \\
(40.18) \\
\end{array}$ & $\begin{array}{c}38.66 \\
(62.00) \\
\end{array}$ & $\begin{array}{c}40.16 \\
(39.30) \\
\end{array}$ \\
\hline $\mathrm{D}_{7}-24^{\text {th }}$ July 2010 & $\begin{array}{c}45.00 \\
(42.11) \\
\end{array}$ & $\begin{array}{c}43.00 \\
(40.95) \\
\end{array}$ & $\begin{array}{r}44.00 \\
(41.53) \\
\end{array}$ \\
\hline \multirow[t]{2}{*}{ Mean } & $\begin{array}{c}31.28 \\
(33.99) \\
\end{array}$ & $\begin{array}{c}28.76 \\
(32.41) \\
\end{array}$ & \\
\hline & \multicolumn{2}{|c|}{ S.Em. \pm} & C.D .at $5 \%$ \\
\hline Sowing dates (D) & \multicolumn{2}{|c|}{1.21} & 3.50 \\
\hline Hybrids $(\mathrm{H})$ & \multicolumn{2}{|c|}{0.64} & 1.85 \\
\hline $\operatorname{Interaction}(\mathrm{D} \times \mathrm{H})$ & \multicolumn{2}{|c|}{1.71} & 4.95 \\
\hline
\end{tabular}

Figures in parentheses indicate Angular transformed values. 
Evaluation of commonness of earhead caterpillars by changing sowing dates in kharif Sorghum

Table 3: Effects of different dates of sowing on the Grain yield of kharif sorghum

\begin{tabular}{|c|c|c|c|}
\hline \multirow{2}{*}{ Dates of sowing } & \multicolumn{3}{|c|}{ Yield (q/ha) } \\
\hline & CSH-16 & CSH-14 & Mean \\
\hline $\mathrm{D}_{1}-12^{\text {th }}$ June 2010 & 37.96 & 39.81 & 38.88 \\
\hline $\mathrm{D}_{2}-19^{\text {th }}$ June 2010 & 39.00 & 39.78 & 39.39 \\
\hline $\mathrm{D}_{3}-27^{\text {th }}$ June 2010 & 36.22 & 38.04 & 37.13 \\
\hline $\mathrm{D}_{4}-03^{\text {rd }}$ July 2010 & 32.66 & 34.60 & 33.63 \\
\hline $\mathrm{D}_{5}-10^{\text {th }}$ July 2010 & 24.82 & 15.17 & 19.99 \\
\hline $\mathrm{D}_{6}-17^{\text {th }}$ July 2010 & 11.57 & 8.40 & 9.98 \\
\hline $\mathrm{D}_{7}-24^{\text {th }}$ July 2010 & 18.56 & 12.90 & 15.73 \\
\hline \multirow[t]{2}{*}{ Mean } & 28.68 & 26.96 & \\
\hline & \multicolumn{2}{|c|}{ S.Em. \pm} & C.D. at $5 \%$ \\
\hline Sowing dates (D) & \multicolumn{2}{|c|}{1.71} & 4.95 \\
\hline Hybrids $(\mathrm{H})$ & \multicolumn{2}{|c|}{0.91} & 2.63 \\
\hline Interaction $(\mathrm{D} \times \mathrm{H})$ & \multicolumn{2}{|c|}{2.42} & 7.01 \\
\hline
\end{tabular}

